\title{
The improvement of strain estimation using universal kriging
}

\author{
Y. Ghiasi • V. Nafisi
}

Received: 31 May 2014/ Accepted: 20 February 2015/Published online: 4 March 2015

(C) Akadémiai Kiadó 2015

\begin{abstract}
In this paper, universal kriging with linear trend is used to interpolate the strain tensor elements over a region along San Andreas Fault in California. The main goal of this paper is to improve the ordinary kriging interpolation results. A 7-year time series (2006-2012) of 12 permanent stations is utilized to obtain the coordinate changes in UTM coordinates system and calculate the strain tensor elements by means of finite difference method. Comparing the results we can find an improvement about $40 \%$ for universal kriging at critical points in which ordinary kriging can't be appropriate method of interpolation.
\end{abstract}

Keywords Strain · Ordinary kriging · Universal kriging · Linear model

\section{Introduction}

By advancement of satellite geodesy, the strain tensor can be estimated by the assistance of coordinates obtained from GPS stations (Burbank and Anderson 2008; Rontogianni 2010; Palano et al. 2013). Although triangulation is one of the most familiar methods of spreading the strain tensor over the desired area, but one cannot decide clearly to which point the strain tensors should be referred. This problem arises from large extent of projections of these elements on the Earth. This fact shows the important role of other methods like finite difference which can calculate the strain tensor point-to-point (Becker and Kaus 2010; Ismail-Zadeh and Tackley 2008). In addition, it's shown that the standard deviation of point-wise methods, in particular finite difference, is less than those of other methods (Bagherifam 2014).

\footnotetext{
Y. Ghiasi · V. Nafisi ( ()

Department of Geomatics Engineering, Faculty of Engineering, University of Isfahan, Esfahān, Iran e-mail: vnafisi@gmail.com; nafisi@eng.ui.ac.ir

Y. Ghiasi

e-mail: yusuf.ghiasi@gmail.com
} 
Therefore having a suitable surface is an important requirement for determination of the strain tensor of arbitrary points. Since the tensor of strain is assumed to be homogenouslike in a desired region, therefore it's common to use deterministic and well-known methods of interpolation such as nearest neighborhood, linear, and sometimes spline or polynomial surfaces (Ardalan and Raoofian 2008; Mashhadi Hossainali et al. 2010; Palano et al. 2013; Tong et al. 2012). But geostatistical methods of interpolation such as ordinary kriging can produce more accurate results.

Combination of various constraints with the specific mathematical equations can be considered as a method to improve the strain estimation results. This technique has been widely studied during past two decades. Kreemer et al. (2003) constructed a global model for horizontal velocity and horizontal strain rate over major plate boundaries. Within their research the velocity field was derived through a least-squares interpolation method using bicubic Bessel spline. Freed et al. (2007) found the relation between the earthquakes and the stress changes and accordingly, other effective factors were interfered. One of the simplest kinds of these constraints is the trend of the strain tensor that can be considered linear or of higher degree polynomials. For strain estimation in a dense network of stations, the linear model is the best model which is always valid (Ardalan and Raoofian 2008).

The kriging as more reliable interpolation method could be categorized into subdivisions such as simple kriging, ordinary kriging and universal kriging. Ordinary kriging is the simplest and the most commonly used, because no trend is defined to comply with. The universal kriging includes modeling of the local trend through the surrounding stations. This modeling as a smooth function of the coordinates is the most important characteristics of the universal kriging (Goovaerts 1997).Geostatistical methods, especially universal kriging have been increasingly utilized in many disciplines such as mining, meteorology, hydrology, geology, remote sensing, soil science, ecology and environmental science (Chirlin and Dagan 1980; Bastin et al. 1984; Hill and Alexandar 1989; White et al. 1997; Duc et al. 2000). For example, Gundugdu and Guney (2007) applied universal kriging with linear trend for spatial analysis of groundwater level. As mentioned before, strain has a linear trend, therefore in this paper we show the improvement of ordinary kriging by assuming a linear trend which converts it to universal kriging.

\section{The tensor of strain and its linear model}

The strain tensor in three dimensions is a symmetric matrix including nine elements which reduce to four elements in two-dimensional case as follow (Turcotte and Schubert 2002):

$$
\varepsilon=\left[\begin{array}{ll}
\varepsilon_{x x} & \varepsilon_{x y} \\
\varepsilon_{y x} & \varepsilon_{y y}
\end{array}\right]
$$

where $\varepsilon_{x x}$ and $\varepsilon_{y y}$ are the normal strain or strain in $\mathrm{x}$ and $\mathrm{y}$ directions respectively, and $\varepsilon_{x y}=\varepsilon_{y x}$ indicate shear strain. It's common to define other parameters based on eigenvalues of this tensor which are obviously invariant with respect to changes in coordinate system, for example (Turcotte and Schubert 2002):

$$
\begin{gathered}
\text { Dilation }=\varepsilon_{x}+\varepsilon_{y} \\
\text { MaxShear }=\max \{\text { EigenValues }\}-\min \{\text { EigenValues }\}
\end{gathered}
$$


As mentioned before, it's preferred to utilize linear model for strain estimation if the network of node points is assumed to be dense. It means that displacements can be modeled as follow:

$$
\begin{aligned}
& \Delta x=a_{0}+a_{1} x+a_{2} y \\
& \Delta y=b_{0}+b_{1} x+b_{2} y,
\end{aligned}
$$

where $\Delta x$ and $\Delta y$ are displacements in $\mathrm{x}$ and $\mathrm{y}$ directions and the coefficients form a matrix as follow:

$$
\mathrm{E}=\left[\begin{array}{ll}
a_{1} & a_{2} \\
b_{1} & b_{2}
\end{array}\right]
$$

Considering basic definition of strain as spatial variation or gradient of displacement, one can find:

$$
\varepsilon=\frac{1}{2}\left(E+E^{T}\right)
$$

This is the desired linear function of coordinates which should be considered in universal kriging.

\section{Models}

Since weighting procedure in geostatistical methods, especially kriging, depends on both distance and statistical distribution, therefore the method of variography is used to estimate the weight. Variography is a term which refers to fitting a spatial model to data and its implementation leads to semivariance as follow (Borrough 1986):

$$
\gamma(h)=\frac{1}{2 N} \sum_{i=1}^{N}\left[Z\left(x_{i}+h\right)-Z\left(x_{i}\right)\right]^{2},
$$

where $\gamma$ is the variogram, $h$ is the distance between two desired points $x_{i}$ and $x_{j}\left(=x_{i}+h\right)$, $N$ is the number of all points, $Z$ is the value at each point. If the mean is assumed to be constant, for example in ordinary kriging which will be discussed later, it can be concluded that:

$$
\operatorname{Var}\left[Z\left(S_{i}\right)-Z\left(S_{j}\right)\right]=2 \gamma_{i j}
$$

The semivariance is half of the variance of the differences between all possible points with a constant distance apart. For semivariance we can define different models i.e. spherical, exponential and Gaussian. For example exponential model which is used in this paper is formulated as follow (Borrough 1986):

$$
\gamma(h)=c\left(1-\exp \left(-\frac{h}{a}\right)\right)
$$

where $c$ and $a$ are sill and range of the semivariogram, respectively. 


\subsection{Ordinary kriging}

The ordinary kriging model assumption is (Goovaerts 1997):

$$
Z\left(S_{i}\right)=\mu+\delta\left(S_{i}\right),
$$

where $\mu$ is the constant mean of the model and $\delta$ is a zero mean stochastic term with variogram $2 \gamma$. Also the predictor assumption is (Goovaerts 1997):

$$
\widehat{Z}\left(S_{0}\right)=\sum_{i=1}^{N} \omega_{i} Z\left(S_{i}\right),
$$

where $S_{0}$ is a point which its value $\widehat{Z}\left(S_{0}\right)$ is going to be predicted, $S_{i}$ is the node point which its value $Z\left(S_{i}\right)$ is used to predict the value of $S_{0}$ and $\omega_{i}$ is the weight of the related point, subject to the constraint $\sum_{i=1}^{N} \omega_{i}=1$ to ensure unbiasedness.

Ordinary kriging minimizes the mean squared error of prediction. It means that:

$$
\min \sigma_{p}^{2}=E\left[Z\left(S_{0}\right)-\widehat{Z}\left(S_{0}\right)\right]^{2}=E\left[Z\left(S_{0}\right)-\sum_{i=1}^{N} \omega_{i} Z\left(S_{i}\right)\right]^{2}
$$

By expanding $\sigma_{p}^{2}$ and differentiating it with respect to $\omega_{i}$, it would be possible to obtain $\omega_{i}$ for all points of the model:

$$
\begin{aligned}
& {\left[Z\left(S_{0}\right)-\sum_{i=1}^{N} \omega_{i} Z\left(S_{i}\right)\right]^{2}=Z^{2}\left(S_{0}\right)-2 Z\left(S_{0}\right) \sum_{i=1}^{N} \omega_{i} Z\left(S_{i}\right)+\sum_{i=1}^{N} \sum_{j=1}^{N} \omega_{i} \omega_{j} Z\left(S_{i}\right) Z\left(S_{j}\right)} \\
& =\sum_{i=1}^{N} \omega_{i} Z^{2}\left(S_{0}\right)-2 \sum_{i=1}^{N} \omega_{i} Z\left(S_{0}\right) Z\left(S_{i}\right)+\sum_{i=1}^{N} \sum_{j=1}^{N} \omega_{i} \omega_{j} Z\left(S_{i}\right) Z\left(S_{j}\right) \\
& \quad-\frac{1}{2} \sum_{i=1}^{N} \omega_{i} Z^{2}\left(S_{i}\right)-\frac{1}{2} \sum_{j=1}^{N} \omega_{j} Z^{2}\left(S_{j}\right)+\sum_{i=1}^{N} \omega_{i} Z^{2}\left(S_{i}\right) \\
& =-\frac{1}{2} \sum_{i=1}^{N} \sum_{j=1}^{N} \omega_{i} \omega_{j}\left[Z\left(S_{i}\right)-Z\left(S_{j}\right)\right]^{2}+\sum_{i=1}^{N} \omega_{i}\left[Z\left(S_{0}\right)-Z\left(S_{i}\right)\right]^{2}
\end{aligned}
$$

By applying expectation on the last expression, it will be:

$$
\begin{aligned}
& -\frac{1}{2} \sum_{i=1}^{N} \sum_{j=1}^{N} \omega_{i} \omega_{j} E\left[Z\left(S_{i}\right)-Z\left(S_{j}\right)\right]^{2}+\sum_{i=1}^{N} \omega_{i} E\left[Z\left(S_{0}\right)-Z\left(S_{i}\right)\right]^{2} \\
& -\frac{1}{2} \sum_{i=1}^{N} \sum_{j=1}^{N} \omega_{i} \omega_{j} \operatorname{Var}\left[Z\left(S_{i}\right)-Z\left(S_{j}\right)\right]+\sum_{i=1}^{N} \omega_{i} \operatorname{Var}\left[Z\left(S_{0}\right)-Z\left(S_{i}\right)\right]
\end{aligned}
$$

But $\operatorname{Var}\left[Z\left(S_{i}\right)-Z\left(S_{j}\right)\right]=2 \gamma_{i j}$ is the definition of the variogram. Therefore ordinary kriging minimizes 


$$
\begin{array}{r}
\sigma_{p}^{2}=E\left[Z\left(S_{0}\right)-\sum_{i=1}^{N} \omega_{i} Z\left(S_{i}\right)\right]^{2}=-\sum_{i=1}^{N} \sum_{j=1}^{N} \omega_{i} \omega_{j} \gamma_{i j}+2 \sum_{i=1}^{N} \omega_{i} \gamma_{i 0} \\
\text { Subject to } \sum_{i=1}^{N} \omega_{i}=1
\end{array}
$$

The minimization is carried out over $\omega_{i}$ subject to non-biased constraint. Therefore this minimization calls for the definition of a Lagrangian $L(u)$ which is a function of the data weights $\omega_{i}$ and Lagrange parameter $2 \lambda$. Therefore the minimization problem can be written as (Edwards and Penney 1982):

$$
\min 2 \sum_{i=1}^{N} \omega_{i} \gamma_{i 0}-\sum_{i=1}^{N} \sum_{j=1}^{N} \omega_{i} \omega_{j} \gamma_{i j}-2 \lambda\left(\sum_{i=1}^{N} \omega_{i}-1\right)
$$

After differentiating with respect to $\omega_{i}$ and $\lambda$ and setting the derivatives equal to zero, it yields:

$$
\left\{\begin{array}{c}
\sum_{i=1}^{N} \omega_{i} \gamma_{i j}-\lambda=\gamma_{i 0} \\
\sum_{i=1}^{N} \omega_{i}=1
\end{array}\right.
$$

and in matrix form:

$$
\Gamma W=C,
$$

where

$$
\begin{gathered}
W=\left[\omega_{1} \omega_{2} \ldots \omega_{n} \lambda\right]^{T} \\
C=\left[\gamma_{10} \gamma_{20} \ldots \gamma_{n 0} 1\right]^{T} \\
\Gamma=\left[\begin{array}{cccccc}
\gamma_{11} & \gamma_{12} & \gamma_{13} & \cdots & \gamma_{1 n} & 1 \\
\gamma_{21} & \gamma_{22} & \gamma_{23} & \cdots & \gamma_{2 n} & 1 \\
\vdots & \vdots & \vdots & \vdots & \vdots & \vdots \\
\gamma_{n 1} & \gamma_{n 2} & \gamma_{n 3} & \cdots & \gamma_{n n} & 1 \\
1 & 1 & 1 & 1 & 1 & 0
\end{array}\right]
\end{gathered}
$$

Therefore the weights $\omega_{i}$ and Lagrange multiplier can be obtained by $W=\Gamma^{-1} C$.

\subsection{Universal kriging}

The ordinary kriging algorithm implicitly considers a non-stationary random function, but in some situations it is not reasonable to consider the local mean $\mu$ as a constant value. In other words, the mean can be a function of the coordinates X, Y, in linear, quadratic, or higher form. Considering a linear trend for strain as follow:

$$
\widehat{Z}\left(S_{i}\right)=\beta_{0}+\beta_{1} X_{i}+\beta_{2} Y_{i}+\delta\left(S_{i}\right)
$$


The value of $\widehat{Z}\left(S_{i}\right)$ can be expressed as:

$$
\begin{array}{rr}
\widehat{Z}\left(S_{i}\right)= & \sum_{i=1}^{N} \omega_{i} Z\left(S_{i}\right)=\sum_{i=1}^{N} \omega_{i} \beta_{0}+\sum_{i=1}^{N} \omega_{i} \beta_{1} X_{i}+\sum_{i=1}^{N} \omega_{i} \beta_{2} Y_{i}+\sum_{i=1}^{N} \omega_{i} \delta\left(S_{i}\right) \\
= & \beta_{0}+\beta_{1} \sum_{i=1}^{N} \omega_{i} X_{i}+\beta_{2} \sum_{i=1}^{N} \omega_{i} Y_{i}+\sum_{i=1}^{N} \omega_{i} \delta\left(S_{i}\right)
\end{array}
$$

which means three constraints of unbiasedness versus one constraint of ordinary kriging:

$$
\sum_{i=1}^{N} \omega_{i} X_{i}=X_{0} ; \sum_{i=1}^{N} \omega_{i} Y_{i}=Y_{0} ; \sum_{i=1}^{N} \omega_{i}=1
$$

By knowing the trend, now the weighting procedure in universal kriging would be similar to ordinary kriging which is done by minimization of the mean squared error of prediction, $\sigma_{p}^{2}$. Using matrix notation, the system of equations will be:

$$
\begin{aligned}
& W=\left[\begin{array}{lll}
\omega_{1} \omega_{2} & \ldots & \omega_{n} \lambda_{1} \lambda_{2} \lambda_{3}
\end{array}\right]^{T} \\
& C=\left[\begin{array}{llll}
\gamma_{10} \gamma_{20} & \ldots & \gamma_{n 0} X_{0} Y_{0} & 1
\end{array}\right]^{T} \\
& \Gamma=\left[\begin{array}{ccccccccc}
\gamma_{11} & \gamma_{12} & \gamma_{13} & \ldots & \ldots & \gamma_{1 n} & X_{1} & Y_{1} & 1 \\
\gamma_{21} & \gamma_{22} & \gamma_{23} & \ldots & \ldots & \gamma_{2 n} & X_{2} & Y_{2} & 1 \\
& & & & \vdots & & & & \\
\gamma_{n 1} & \gamma_{n 2} & \gamma_{n 3} & \ldots & \ldots & Y_{n n} & X_{n} & Y_{n} & 1 \\
X_{1} & X_{2} & X_{3} & \ldots & \ldots & X_{n} & 0 & 0 & 0 \\
Y_{1} & Y_{2} & Y_{3} & \ldots & \ldots & Y_{n} & 0 & 0 & 0 \\
1 & 1 & 1 & \ldots & \ldots & 1 & 0 & 0 & 0
\end{array}\right]
\end{aligned}
$$

\section{Numerical results}

A numerical comparison between ordinary kriging and universal kriging interpolations was carried out by downloading the time series of 12 permanent GPS stations from http://sideshow. jpl.nasa.gov/post/series.html which are located along southern part of San Andreas Fault. This time series is collected in a 7-year interval from 2005 to 2012 (2183 observational day) and its stations are named P210, P217, P212, P233, P234, P236, P238, P240, P242, P244, P251 and $\mathrm{P} 239$. The coordinates and the strain tensor of network points are tabulated in Table 1 and the map of these stations is shown in Fig. 1. Strain tensor is calculated for each point through finite difference method. Then, the strain tensor is interpolated through four methods of linear, spline, ordinary kriging and universal kriging. The results are tabulated in Table 2. It can be noticed that linear and spline interpolations are able to interpolate only points located inside the desired region and for marginal points it is not possible to calculate the values.

Talking about the details of kriging interpolation, it can be paid attention that the semivariogram is assumed to be exponential; because these methods are very similar to each other and there is no significant difference between various models of semivariogram in strain interpolation, e.g. spherical, exponential, Gaussian. Also, as mentioned before, there is a constant value in kriging, which is named as sill of the semivariogram. Estimating this constant value might be done by difference methods e.g. ordinary least squares, maximum likelihood, by-eye methods, etc. In this paper, the value of $c$ is tested by-eye, so 


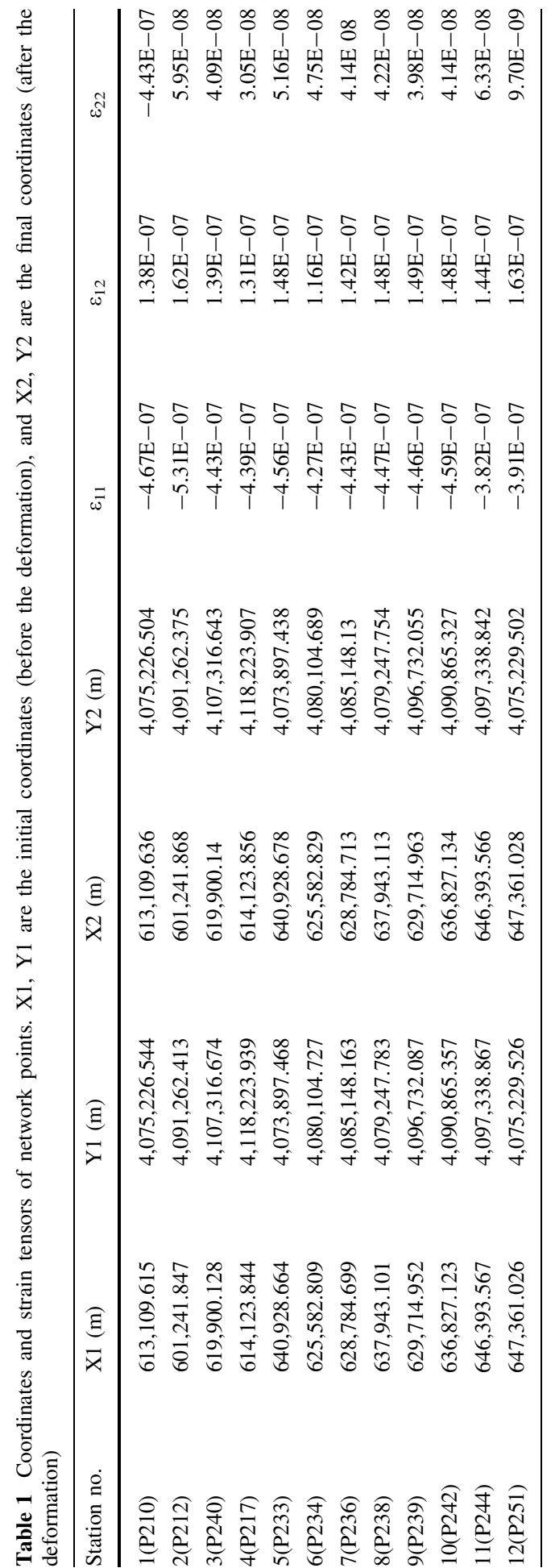



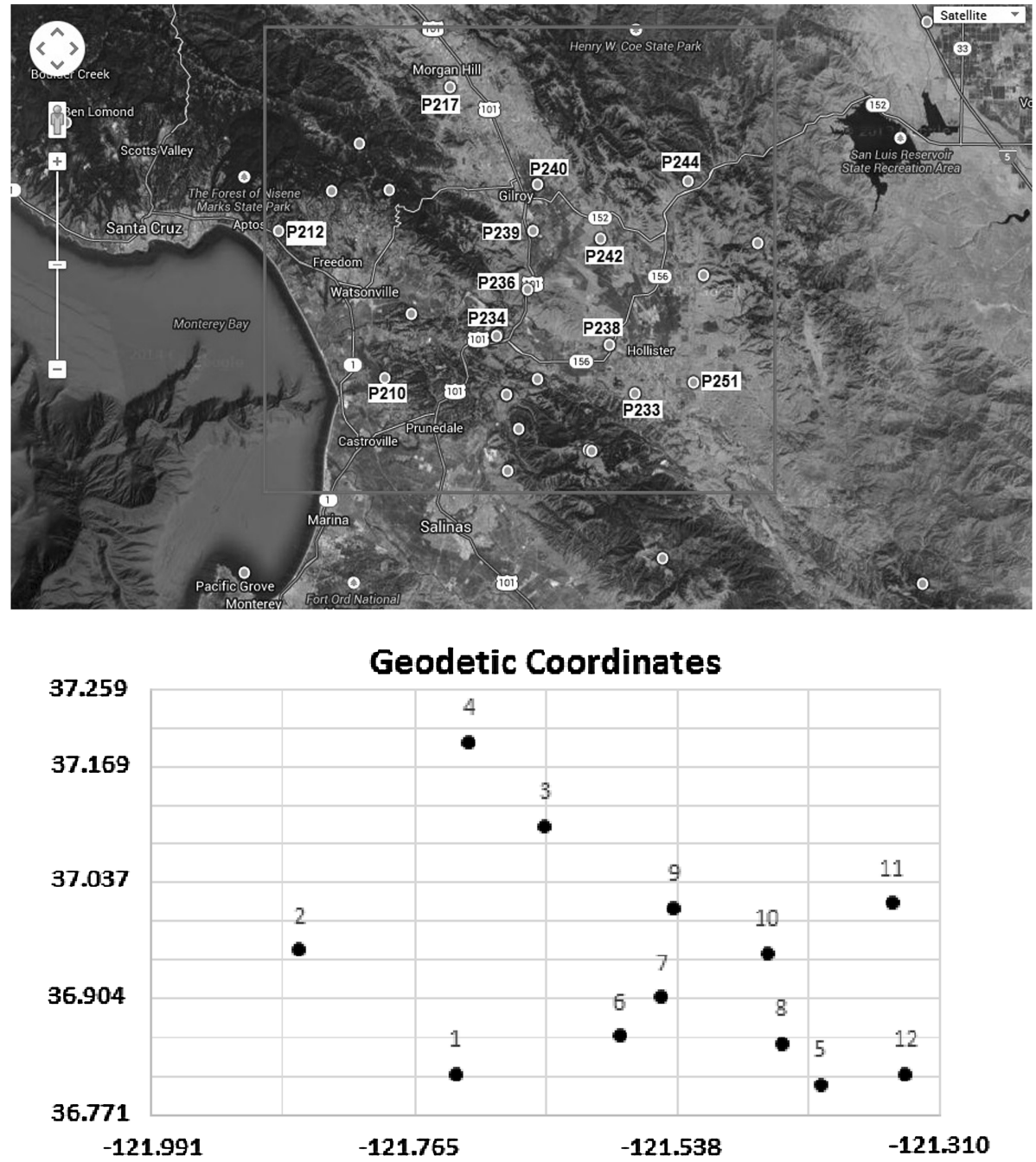

Fig. 1 Up location of 12 stations of study on the Earth map. Down geodetic coordinates of 12 station

that the average of the error percentages of interpolated points are tested for a huge number of sill values and the result of this comparison is graphed in Fig. 2. As shown in this graph the value of the variogram sill, $c$ is preferred to be one for ordinary kriging and 8.9 for universal kriging to reduce the error percentage average for all interpolated points.

Then validation procedure is implemented by excluding each point respectively and recalculating its strain tensor through the other strain points by means of ordinary kriging and universal kriging interpolation and comparing the discrepancies between calculated and predicted strain tensors. It should be noticed that by excluding each point, variogram is estimated again for remaining points. Results are shown in Table 2 and Fig. 3. All of the calculations are done and programmed in MATLAB.

As it can be seen, kriging interpolation methods, either ordinary or universal, provide acceptable results for $\varepsilon_{11}$ and $\varepsilon_{22}$ and on average, we can find improvements more than 
Table 2 Error percentage of kriging, linear and spline for network points

\begin{tabular}{|c|c|c|c|c|c|c|c|c|c|c|c|c|}
\hline \multirow[t]{2}{*}{ Station no. } & \multicolumn{3}{|c|}{ Ordinary kriging } & \multicolumn{3}{|c|}{ Universal kriging } & \multicolumn{3}{|l|}{ Linear } & \multicolumn{3}{|l|}{ Spline } \\
\hline & $\varepsilon_{11}$ & $\varepsilon_{12}$ & $\varepsilon_{22}$ & $\varepsilon_{11}$ & $\varepsilon_{12}$ & $\varepsilon_{22}$ & $\varepsilon_{11}$ & $\varepsilon_{12}$ & $\varepsilon_{22}$ & $\varepsilon_{11}$ & $\varepsilon_{12}$ & $\varepsilon_{22}$ \\
\hline 1 & - & - & - & - & - & - & - & - & - & - & - & - \\
\hline 2 & - & - & - & - & - & - & - & - & - & - & - & - \\
\hline 3 & 0.14 & 7.64 & 3.24 & 1.63 & 3.05 & 8.60 & 21.4 & 41.91 & 183.68 & 22.36 & 44.15 & 172.73 \\
\hline 4 & - & - & - & - & - & - & - & - & - & - & - & - \\
\hline 5 & - & - & - & - & - & - & - & - & - & - & - & - \\
\hline 6 & 0.83 & 6.55 & 2.94 & 3.98 & 0.41 & 6.25 & 21.44 & 5.04 & 67.35 & 19.4 & 0.85 & 73.58 \\
\hline 7 & 0.32 & 6.56 & 1.42 & 1.67 & 11.08 & 4.76 & 19.72 & 11.44 & 90.91 & 20.43 & 15.81 & 94.59 \\
\hline 8 & 1.19 & 9.39 & 2.71 & 2.28 & 0.90 & 0.03 & 14.24 & 1.72 & 120.52 & 14.7 & 0.5 & 122.92 \\
\hline 9 & 0.72 & 5.50 & 1.88 & 0.91 & 20.90 & 2.46 & 18.91 & 19.72 & 100.54 & 17.86 & 19.6 & 107.36 \\
\hline 10 & 0.67 & 5.83 & 2.43 & 4.40 & 17.87 & 0.07 & 9.51 & 1.04 & 77 & 10.91 & 1.21 & 76.37 \\
\hline 11 & - & - & - & - & - & - & - & - & - & - & - & - \\
\hline 12 & - & - & - & - & - & - & - & - & - & - & - & - \\
\hline
\end{tabular}
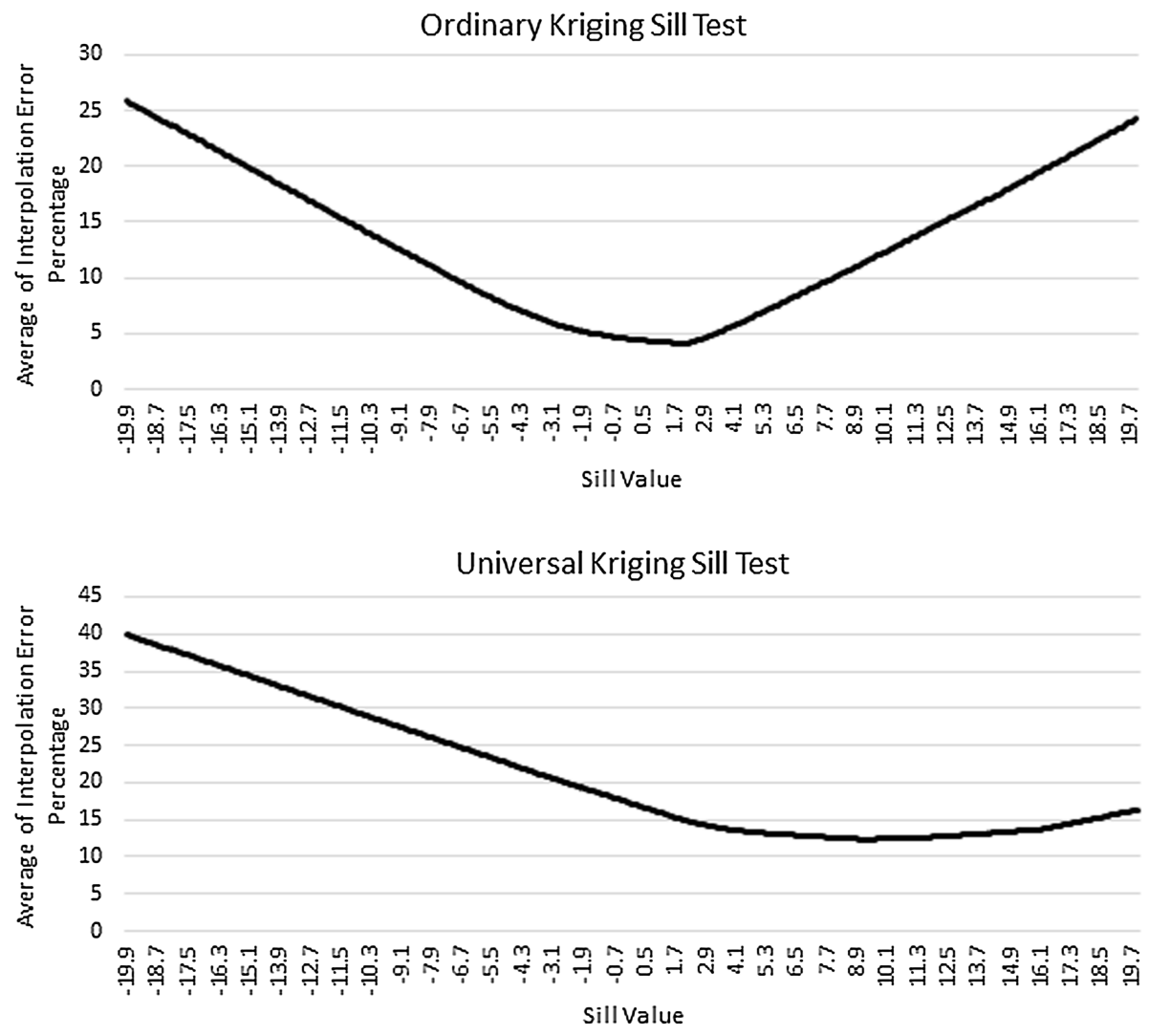

Fig. 2 Testing the value of the sill of the variogram for ordinary and universal kriging 


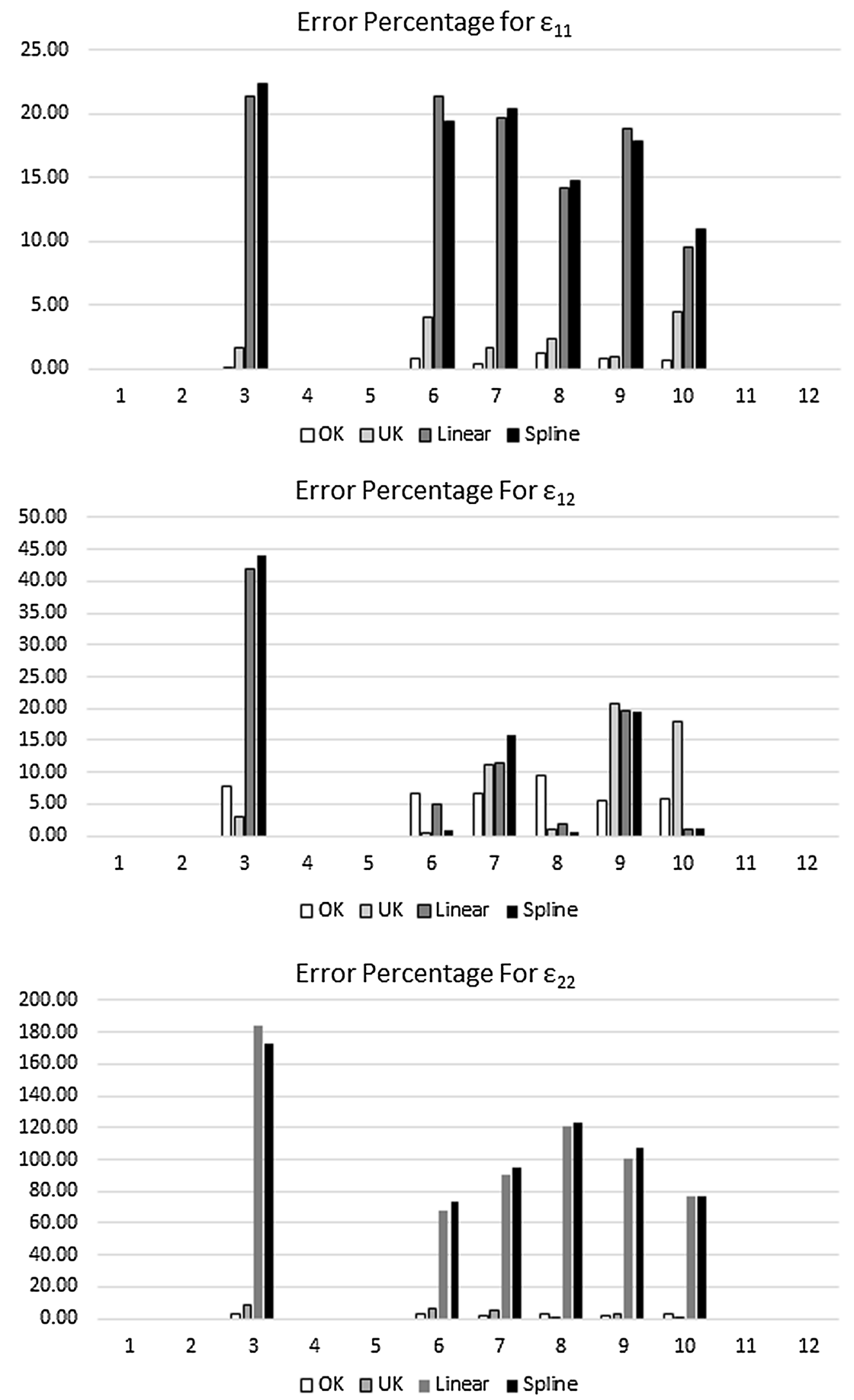

Fig. 3 The error percentage of kriging, linear and spline for each point. Vertical axis is the error percentage and horizontal axis is the number of each point 
$70 \%$ with respect to results of linear and spline interpolations. But the critical case is estimation of shear strain $\varepsilon_{12}$; the performance of ordinary kriging is better than linear and spline, only in 3 point, but its performance on points 6,8 and 10 is not acceptable. Since linear interpolation is one of the most usual method for strain calculations (Ardalan and Raoofian 2008) therefore a linear trend is combined with ordinary kriging, as explained in 3.2 , and estimate $\varepsilon_{12}$ again. Results show that universal kriging error percentage for $\varepsilon_{12}$ is usually equal or less than of linear interpolation which are acceptable. In other words, universal kriging can improve estimation of shear strain.

\section{Conclusions and recommendations}

The results of ordinary kriging interpolation for strain estimation show an improvement of about $70 \%$ with respect to results of linear and spline method. But it has some deficiencies and disadvantages. For example it is not sensitive to introducing trend in the data over the study area, whereas it is known that strain has almost a linear trend. Therefore by adding linear trend to ordinary kriging and employing universal kriging for strain estimation, we can find about $40 \%$ improvement especially for shear strain element.

It should be noticed that we can choose non-linear trend according to the condition of the case study area and also the number of unbiased constraint can differ and should be considered in the weight matrices.

\section{References}

Ardalan AA, Raoofian M (2008) A proposal for deformation analysis via direct computation of strain tensor elements from the time-wise changes in the distances and angles in a geodetic network; case study: deformation computation of the geodynamic network of Iran. J Phys Earth Space 35(2):37-60 (In Persian)

Bagherifam T (2014) Investigation of strain analysis method validity in geodynamics displacements using GNSS data. Master Theses. University of Isfahan, Isfahan (In Persian)

Bastin G, Lorent B, Duque C, Gevers M (1984) Optimal estimation of the average rainfall and optimal selection of raingage locations. Water Resour Res 20(4):463-470

Becker TW, Kaus BJP (2010) Numerical geodynamics; an introduction to computational methods with focus on solid earth applications of continuum mechanics. University of Southern California, Los Angeles

Borrough PA (1986) Principles of geographical information systems for land resources assessment. Oxford University Press, New York

Burbank DW, Anderson RS (2008) Tectonic geomorphology, 7th edn. Balckwell Science, Malden, Oxford, Carlton

Chirlin GR, Dagan G (1980) Theoritical head variogram for steady flow in statistically homogeneous aquifers. Water Resour Res 16(6):1001-1015

Duc H, Shannon I, Azzi M (2000) Spatial distribution characteristics of some air pollutants in Sydney. Math Comput Simul 54(1-3):1-21

Edwards CH, Penney DE (1982) Calculus and analytic geometry. Prentice-Hall, Englewood Cliffs, pp 146-149

Freed AM, Ali ST, Burgmann R (2007) Evolution of stress in Southern California for the past 200 years from coseismic, postseismic and interseismic stress changes. Geophys J Int 169(3):1164-1179

Goovaert P (1997) Geostatistics for natural resources evaluation: applied geostatitistics series. Oxford University, Oxford

Gundugdu KS, Guney I (2007) Spatial analyses of groundwater levelsusing universal kriging. J Earth Syst Sci 116(1):49-55

Hill M, Alexandar F (1989) Statistical methods used in assessing the risk of disease near a source of possible environmental pollution: a review. J R Stat Soc 152:353-363 
Ismaeil-Zadeh A, Tackley P (2008) Computational method for geodynamics. Moscow, Cambridge

Kreemer C, Holt WE, Haines AJ (2003) An integrated global model of present-day plate motions and plate boundary deformation. Geophys J Int 154(1):8-34

Mashhadi Hossainali M, Becker M, Groten E (2010) Comprehensive approach to the analysis of the 3D kinematics deformation with application to the Kenai Peninsula. J Geod Sci 1(1):59-73

Palano M, Imprescia P, Gresta S (2013) Currentstressandstrain-rate fields across the Dead Sea fault system: constraints from seismological data and GPS observations. Earth Planet Sci Lett 369-370:305-316

Rontogianni S (2010) Comparison of geodetic and seismic strain rates in Greece by using a uniform processing approach to campaign GPS measurements over the interval 1994-2000. J Geodyn 50:381-399

Tong X, Sandwell DT, Smith-Konter B (2012) High-resolution interseismic velocity data along the San Andreas Fault from GPS and InSAR. J Geophys 118:1-21

Turcott DL, Schubert G (2002) Geodynamics, 2nd edn. Cambridge University Press, Cambridge

White JG, Welch RM, Norvell WA (1997) Soil ZincMap of the USA using geostatistics and geographic information systems. Soil Sci Soc Am J 61(1):185-194 